\title{
Cancer survival in China, 2003-2005: A population-based study
}

Hongmei Zeng ${ }^{1^{\star}}$, Rongshou Zheng ${ }^{1^{\star}}$, Yuming Guo ${ }^{2}$, Siwei Zhang ${ }^{1}$, Xiaonong Zou ${ }^{1}$, Ning Wang ${ }^{3}$, Limei Zhang ${ }^{4}$, Jingao Tang ${ }^{5}$, Jianguo Chen ${ }^{6}$, Kuangrong $\mathrm{Wei}^{7}$, Suqin Huang ${ }^{8}$, Jian Wang ${ }^{9}$, Liang $\mathrm{Yu}^{10}$, Deli Zhao ${ }^{11}$, Guohui Song ${ }^{12}$, Jianshun $\mathrm{Chen}^{13}$, Yongzhou Shen ${ }^{14}$, Xiaoping Yang ${ }^{15}$, Xiaoping Gu ${ }^{16}$, Feng Jin ${ }^{17}$, Qilong $\mathrm{Li}^{18}$, Yanhua $\mathrm{Li}^{19}$, Hengming Ge ${ }^{20}$, Fengdong Zhu ${ }^{21}$, Jianmei Dong ${ }^{22}$, Guoping Guo ${ }^{23}$, Ming Wu ${ }^{24}$, Lingbin $\mathrm{Du}^{25}$, Xibin Sun ${ }^{26}$, Yutong He ${ }^{27}$, Michel P Coleman ${ }^{28}$, Peter Baade ${ }^{29 \dagger}$, Wanqing Chen ${ }^{1 \dagger}$ and Xue Qin Yu ${ }^{30 \dagger}$

${ }^{1}$ National Office for Cancer Prevention and Control, National Cancer Center, Beijing, China

${ }^{2}$ School of Population Health, University of Queensland, Brisbane, Australia

${ }^{3}$ Beijing Office for Cancer Prevention and Control, Peking University Cancer Hospital and Institute, Beijing, China

${ }^{4}$ Dalian Cancer Registry, Dalian Center for Disease Control and Prevention, Liaoning, China

${ }^{5}$ Haimen Cancer Registry, Haimen Center for Disease Control and Prevention, Jiangsu, China

${ }^{6}$ Qidong Cancer Registry, Qidong Liver Cancer Institute, Jiangsu, China

${ }^{7}$ Zhongshan Cancer Registry, Zhongshan People's Hospital, Guangdong, China

${ }^{8}$ Taixing Cancer Registry, Taixing Center for Disease Control and Prevention, Jiangsu, China

${ }^{9}$ Jianhu Cancer Registry, Jianhu Center for Disease Control and Prevention, Jiangsu, China

${ }^{10}$ Linzhou Cancer Registry, Linzhou Cancer Hospital, Henan, China

${ }^{11}$ Feicheng Cancer Registry, Feicheng People's Hospital, Shandong, China

${ }^{12}$ Cixian Cancer Registry, Cixian Institute for Cancer Prevention and Control, Hebei, China

${ }^{13}$ Changle Cancer Registry, Changle Institute for Cancer Prevention and Control, Fujian, China

${ }^{14}$ Haining Cancer Registry, Haining Institute for Cancer Prevention and Control, Zhejiang, China

${ }^{15}$ Jintan Cancer Registry, Jintan Center for Disease Control and Prevention, Jiangsu, China

${ }^{16}$ Dafeng Cancer Registry, Dafeng Center for Disease Control and Prevention, Jiangsu, China

${ }^{17}$ Ganyu Cancer Registry, Ganyu Center for Disease Control and Prevention, Jiangsu, China

${ }^{18}$ Jiashan Cancer Registry, Jiashan Institute for Cancer Prevention and Control, Zhejiang, China

${ }^{19}$ Sihui Cancer Registry, Sihui Institute for Cancer Prevention and Control, Guangdong, China

${ }^{20}$ Donghai Cancer Registry, Donghai Center for Disease Control and Prevention, Jiangsu, China

${ }^{21}$ Guanyun Cancer Registry, Guanyun Center for Disease Control and Prevention, Jiangsu, China

${ }^{22}$ Lianyungang Cancer Registry, Lianyungang Center for Disease Control and Prevention, Jiangsu, China

${ }^{23}$ Yangzhong Cancer Registry, Yangzhong Center for Disease Control and Prevention, Jiangsu, China

${ }^{24}$ Jiangsu Center for Disease Control and Prevention, Jiangsu, China

${ }^{25}$ Zhejiang Office for Cancer Prevention and Control, Zhejiang Cancer Institute, Zhejiang, China

${ }^{26}$ Henan Office for Cancer Prevention and Control, Henan Cancer Hospital, Henan, China

${ }^{27}$ Hebei Office for Cancer Prevention and Control, Fourth Hospital of Hebei Medical University, Hebei, China

${ }^{28}$ Cancer Research UK Cancer Survival Group, London School of Hygiene and Tropical Medicine, London, United Kingdom

${ }^{29}$ Cancer Research Centre, Cancer Council Queensland, Brisbane, Australia

${ }^{30}$ Cancer Research Division, Cancer Council New South Wales, Sydney, Australia

Limited population-based cancer registry data available in China until now has hampered efforts to inform cancer control policy. Following extensive efforts to improve the systematic cancer surveillance in this country, we report on the largest pooled analysis of cancer survival data in China to date. Of 21 population-based cancer registries, data from 17 registries ( $n=138,852$ cancer records) were included in the final analysis. Cases were diagnosed in 2003-2005 and followed until the end of 2010. Age-standardized relative survival was calculated using region-specific life tables for all cancers combined and

Key words: population-based study, cancer registry, relative survival, geographical disparity, China

Abbreviations: CI: confidence intervals; NCCR: the National Central Cancer Registry

${ }^{\star}$ H.Z. and R.Z. contributed equally to the study.

${ }^{\dagger}$ P.B., W.C. and X.Q.Y. (senior authors) contributed equally to the study.

Grant sponsor: Cancer Institute \& Hospital, Chinese Academy of Medical Sciences; Grant number: LC2011Y41; Grant sponsor: Australian NHMRC Career Development Fellowship (P.B.); Grant number: 1005334; Grant sponsor: Australian NHMRC Early Career Fellowship (X.Q.Y.); Grant number: 550002

DOI: $10.1002 / \mathrm{ijc} .29227$

History: Received 9 June 2014; Accepted 27 Aug 2014; Online 20 Sep 2014

Correspondence to: Wanqing Chen, National Office for Cancer Prevention and Control, National Cancer Center, Beijing 100021, China, Tel.: +86108778 7039, Fax: +8610 67718227, E-mail: chenwq@cicams.ac.cn 
26 individual cancers. Estimates were further stratified by sex and geographical area. The age-standardized 5 -year relative survival for all cancers was $30.9 \%$ (95\% confidence intervals: $30.6 \%-31.2 \%)$. Female breast cancer had high survival (73.0\%) followed by cancers of the colorectum ( $47.2 \%)$, stomach $(27.4 \%)$, esophagus $(20.9 \%)$, with lung and liver cancer having poor survival ( $16.1 \%$ and $10.1 \%$ ), respectively. Survival for women was generally higher than for men. Survival for rural patients was about half that of their urban counterparts for all cancers combined ( $21.8 \%$ vs. $39.5 \%)$; the pattern was similar for individual major cancers except esophageal cancer. The poor population survival rates in China emphasize the urgent need for government policy changes and investment to improve health services. While the causes for the striking urban-rural disparities observed are not fully understood, increasing access of health service in rural areas and providing basic health-care to the disadvantaged populations will be essential for reducing this disparity in the future.

\section{What's new?}

Because it's difficult to create good public-health policies without good population data, China has recently made efforts to improve its systematic recording of cancer data. This paper reports the largest pooled analysis of survival data in China, the first to include data from a wide range of geographical areas. They report the various survival rates for different cancers by age, gender, and locality. The most striking finding was that those living in rural residents had far lower survival rates than urban residents. This finding may prompt efforts to improve availability of cancer prevention and treatment in rural areas of China.

Cancer has become a leading cause of death in urban China and the second most common causes in rural China for many years. ${ }^{1}$ The burden of cancer in China is expected to increase as a result of population ageing, increased environmental pollution, uncontrolled chronic infection, and adoption of westernized lifestyles, including tobacco and harmful alcohol use, unhealthy diets and physical inactivity. ${ }^{2}$ Information on the overall effectiveness of cancer management is thus increasingly important. Due to its large population, the global burden of cancer is strongly influenced by the burden of cancer in China. ${ }^{3}$

Population-based cancer registries have provided survival statistics since the 1960s in developed regions such as European countries, ${ }^{4,5}$ while survival estimates have been routinely reported in the United States, Canada and Australia for the last 30 years. ${ }^{6-8}$ Collaborative studies including EUROCARE and CONCORD have provided international comparisons of cancer survival. ${ }^{9,10}$ In China, the low population coverage of cancer registration has historically provided inadequate cancer information. To date, population-based survival statistics have been reported in only a few cancer registries for specific cancers, such as Beijing for breast cancer about 10 years ago, ${ }^{11}$ while only three cancer registries in mainland China were included in an international survival study (2010) initiated by the International Agency for Research on Cancer. ${ }^{12}$

Some cancer registries have existed in China for more than 50 years, but the development of standard cancer registration practice was very slow before the year 2000. This has severely hampered efforts to inform health policy and medical practice to reduce the burden of cancer. In 2002, the National Central Cancer Registry (NCCR) of China was established by the Ministry of Health to enhance systematic management of cancer surveillance. Since then, there has been a noticeable improvement in the coverage and quality of cancer registration. With increasing acknowledgement of the importance of cancer surveillance, 21 cancer registries began collecting the follow-up information required by NCCR in 2011 for surveillance of cancer survival. China now has the capacity to report on cancer survival, and to benchmark outcomes against other countries, thus informing research and health policy priorities in China and internationally.

This study was designed to examine the survival of Chinese cancer patients diagnosed with a first primary cancer during 2003-2005, using standardized quality control and analytical methods. This is the first systematic examination of cancer survival in China using population-based cancer registry data from a wide range of geographical regions.

\section{Material and Methods \\ Cancer cases and registries}

A total of 21 cancer registries provided data. Twenty of these registries provided full data sets for new cancer patients diagnosed during 2003-2005, while one registry (Donghai) only submitted data for patients diagnosed in 2004-2005. All registries had follow-up information for all registered patients on vital status and death from any cause up to 31 December 2010. A mix of active and passive follow-up methods was used to identify the vital status of patients from the date of diagnosis. Passive reporting relies on periodical linkage of cancer registration records with records of death from any cause from the mortality surveillance system in local Centers for Disease Control. Active reporting involves registry personnel examining the sources of data and abstracting the required information on vital status onto special forms from different types of hospital and health insurance systems. 
Home visits or telephone contacts with next of kin were used for patients whose vital status could not be ascertained by other methods. Nineteen registries used both passive and active follow-up. Two registries (Dalian and Haining) used only passive follow-up methods.

The quality and completeness of the cancer registration data was assessed with IARC-crgTools ${ }^{13}$ to identify errors, inconsistencies and unusual combinations of cancer site, morphology, sex and age at diagnosis. Questionable records were sent back to the cancer registry for verification and correction, and records that could not be verified or corrected were excluded. Cancer records were supplied with the anatomical site coded to the tenth revision of the International Classification of Disease (ICD-10). Only the first primary malignant tumour (behavior code 3) in each patient was included. Tumour records were excluded if they were based on a death certificate only, or if the vital status was unknown.

Data from four registries (Donghai, Guanyun, Lianyungang and Yangzhong) were withdrawn, mainly due to incomplete registration for 2003-2005 or incomplete follow-up data. The other 17 cancer registries (shown in Table 1) submitted diagnostic and survival information for all cancer sites and were included in the survival analyses.

\section{Statistical analysis}

We estimated relative survival, the ratio between the survival observed among the cancer patients and the survival that would have been expected if they were only subject to the mortality observed in the general population of the registry's territory. Survival was estimated with the classic cohort approach, in that all patients had at least 5 years of potential follow-up. Observed survival was computed by the life-table method and expected survival was estimated by the Ederer $\mathrm{II}^{14}$ method, using registry-specific life tables by age, sex and single calendar year between 2003 and 2010 . All registries except Beijing provided abridged life tables or raw data on deaths and populations to enable the construction of sex- and year-specific life tables from the local registries. Abridged life tables were smoothed to complete (single-year-of-age) life tables and extended to the age of 99 using the Elandt-Johnson method. ${ }^{15}$ In Beijing and Taixing, linear interpolation ${ }^{16}$ was used to create life tables for the years in which data were missing. Standard errors of relative survival estimates were obtained using Greenwood's formula. ${ }^{17}$

For some analyses we pooled data from urban and rural registries separately. We estimated survival separately for 22 cancers in males and 24 in females. Cancers of the colon and rectum were analyzed together, as were all oral and pharyngeal cancers. The remaining cancers were analyzed as a single group.

To account for differences in the age profile of cancer patients in different parts of China, overall relative survival was adjusted for age with the International Cancer Survival Standard weights. ${ }^{18}$ Five age groups were used for this purpose: $0-44,45-54,55-64,65-74$ and 75-99 years. We classified the areas covered by participating cancer registries as urban or rural, based on standards set by the National Bureau of Statistics in China. ${ }^{19}$ Urban areas (Beijing, Dalian and Zhongshan) are predominately metropolitan cities with high population density, and are more modernized, and technology- and business-driven. The remaining registries were categorized as covering rural areas, which are much less developed and have a more agricultural and farming focus (Fig. 1). We used generalized linear models with a Poisson error structure to calculate the excess hazard ratios (rural $v s$. urban as reference category) of death within 5 years of diagnosis for each type of cancer, including sex and age as covariates. $^{20}$ Descriptive analyses were conducted using SAS (version 9.2, SAS Institute, Cary NC) while survival analyses were performed using strs $^{21}$ in Stata (version 12.1, StataCorp LP, College Station TX).

\section{Results}

The 21 registries, covering a population of over 26.1 million, submitted records for 154,178 cancer patients diagnosed during 2003-2005 (Table 1). Less than 5\% of records were excluded because of major errors $(0.1 \%)$, multiple primary $(0.1 \%)$, death certificate only $(0.6 \%)$, or unknown vital status (3.9\%) and eight records of cases aged 100 years or older. After exclusions, 138,852 patients from 17 registries were included in survival analyses (95.3\% of those eligible). The overall percentage of microscopically verified cases was $65.9 \%$, ranging from $75-81 \%$ in Jintan, Linzhou, Dalian and Zhongshan to $40 \%$ in Sihui.

The four most common cancers (lung, stomach, liver and oesophagus) comprised over half (56.7\%) the 138,852 cases included in the survival analyses (Table 2). Five-year relative survival is below $30 \%$ for each of these cancers, which largely explains the low overall survival for all cancers combined: age-standardized 5-year relative survival 30.9\% [95\% confidence interval (CI) 30.6-31.2\%) for males and females combined, $26.6 \%$ (95\% CI $26.2-27.0 \%$ ) for males and 36.6\% (95\% CI $36.1-37.1 \%$ ) for females.

Five-year survival for the six most common cancers ranged from $10.1 \%$ (95\% CI 9.5-10.7\%) for liver cancer up to $73.0 \%$ (95\% CI $71.2-74.9 \%$ ) for breast cancer in women. Age-standardized 5-year relative survival for cancers of the lung, stomach, oesophagus and colorectum (in reducing order of incidence), pooled for all 17 registries, was 16.1, 27.4, 20.9 and $47.2 \%$, respectively.

Age-standardized 5-year relative survival, pooled for all 17 registries, is presented for 22 cancers in males and 24 in females, and for all cancers combined, in Figure 2. Five-year relative survival was highest for cancers of the breast (females: $73.0 \%)$ and thyroid (67.5\%), and lowest for cancers of the liver $(10.1 \%)$ and pancreas $(11.7 \%)$. Cancers with relatively good prognosis (five-year relative survival $60 \%$ or higher) included cancers of the breast, thyroid, bladder and kidney, comprising about one in eight (12.2\%) of all cancers. 


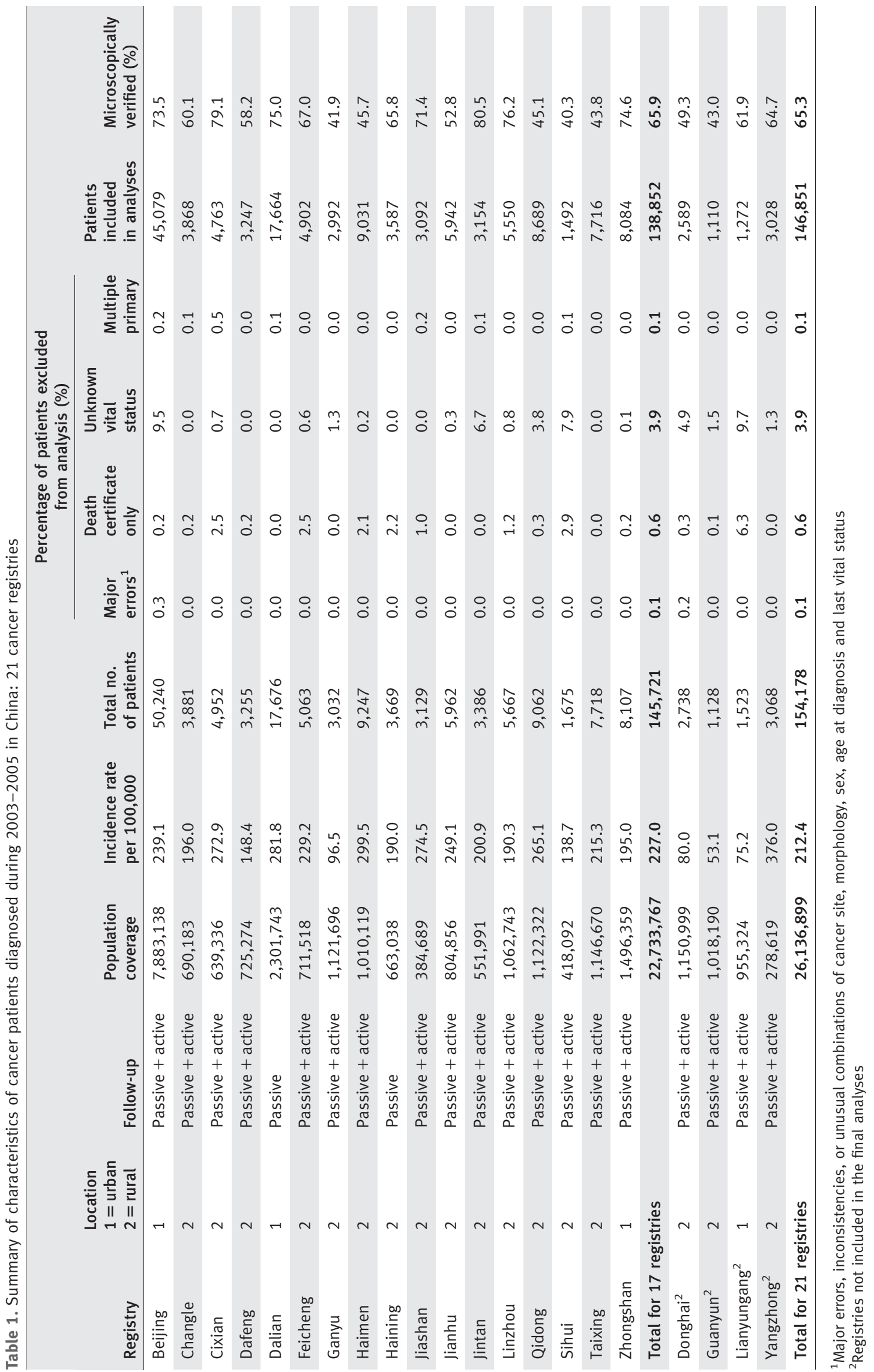


Cancers with poor survival (below 30\%) included cancers of the liver, pancreas, lung, bone, brain, oesophagus, leukaemia, gallbladder and stomach, which collectively account for nearly two-thirds (64.9\%) of all cancer cases.

Survival was markedly lower in rural areas than urban areas, both for all cancers combined and for most individual cancers (Figs. 3 and 4). For all cancers combined, 5-year agestandardized relative survival was $39.5 \%$ (95\% CI 39.1$39.9 \%$ ) in urban areas and $21.8 \%$ (95\% CI $21.4-22.2 \%$ ) in rural areas, equating to an excess hazard ratio of 1.39 (95\% CI 1.38-1.41). The rural deficit in 5-year survival varied by cancer type, with the highest excess hazard ratios in rural areas (vs. urban areas) for cancers of the breast, uterus, kidney and larynx. The excess hazard ratio was significantly greater than unity for 21 of the 27 cancers considered in either sex. The exceptions were other thoracic cancers, cancers of the testis, gallbladder, pancreas and oesophagus-the only cancer for which survival for rural patients was higher than for urban patients (Figs. 3 and 4).

\section{Discussion}

This is the first systematic analysis exploring the overall profile of population-based cancer survival in the Chinese population, with a standardized protocol for data collection, quality control and analysis. The population covered was only about $2 \%$ of the Chinese national population, but this still represents a base population of 26.1 million people. Reliable information on cancer survival in the whole population is critical to measure the overall effectiveness of the diagnosis and management of cancer, including access to efficient treatment, which can then be used by health care planners and professionals. Five-year relative survival in China was generally low compared with that reported from developed countries $^{7,10,22,23}$ and even lower in rural China.

Interpretation of the survival estimates should take into account differences in data quality between the registries. Differences in health awareness and early detection, and the availability, development of and accessibility to cancer treatment within the population should also be considered. ${ }^{24,25}$ In our study, the age-standardized 5-year survival for all cancers combined in China was 30.9\%, much lower than has been reported for developed countries. In the United States, for example, five-year relative survival for all cancers combined was $67.2 \%$ for adults in $2004,{ }^{23}$ and the corresponding Australian estimate (2006-2010) was $66 \%{ }^{22}$ One reason for the differences in cancer survival for all cancers combined between developed countries and China relates to the differences in cancer profile. In China the four most common cancers (lung, stomach, liver, oesophagus), all with 5-year relative survival below $30 \%$, account for $57 \%$ of all cancers, while the corresponding proportion was $18 \%$ in the United States, ${ }^{23}$ and $13 \%$ in Australia. ${ }^{22}$

However, relative survival for most individual cancers was also substantially lower in China than in Australia, Europe and the United States, with the exception of lung cancer, for

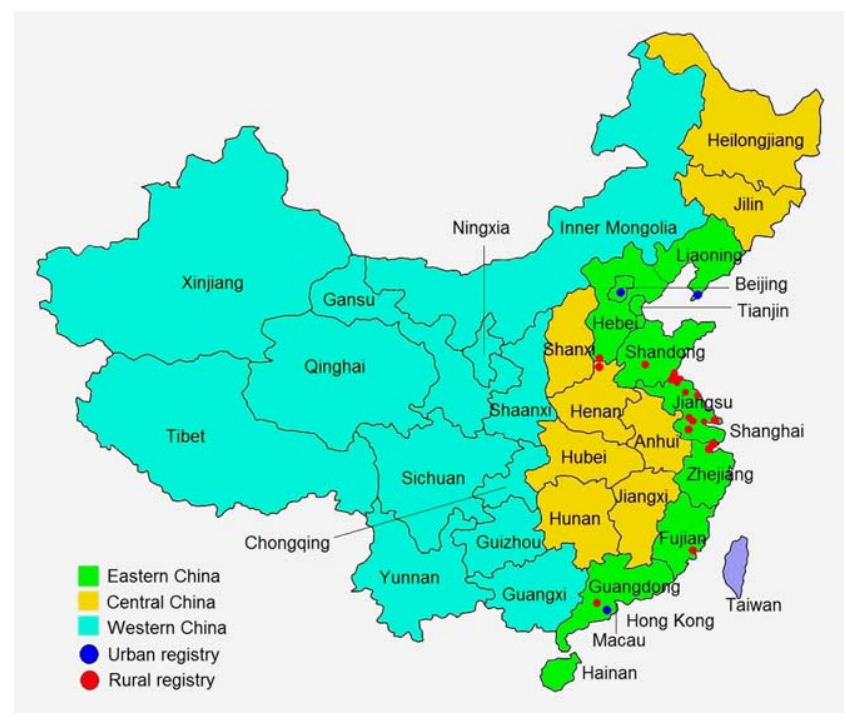

1. Map of the 21 contributing cancer registries and geographic regions in China (The dots are location of the cancer registries).

Geographic regions and their socioeconomic development level in China (mainland)

\begin{tabular}{llll}
\hline $\begin{array}{l}\text { Geographic } \\
\text { region }\end{array}$ & GDP & $\begin{array}{l}\text { \# of Dr \& } \\
\text { nurses per 1,000 }\end{array}$ & $\begin{array}{l}\text { \# of hospital } \\
\text { beds per 1,000 }\end{array}$ \\
\hline Eastern China & US\$5,464 & 5.22 & 3.96 \\
Central China & $\$ 2,630$ & 3.30 & 3.30 \\
Western China & $\$ 2,354$ & 3.76 & 3.35 \\
\hline
\end{tabular}

Figure based on data from the China Health Statistics Yearbook (2011) and (Jian W, Chan KY, Reidpath DD, Xu L. China's ruralurban care gap shrank for chronic disease patients, but inequities persist. Health Aff (Millwood) 2010;29:2189-96.) GDP= gross domestic product

which survival is similar. Minor differences exist in the estimation methods between the various studies cited, but this is unlikely to explain such substantial differences in survival. For breast cancer, the most common cancer in women both in China and developed countries, 5-year survival (73\%) in China was much lower than the corresponding estimates in the United States $(90 \%)^{23}$ and Australia (89\%), ${ }^{22}$ and to a lesser degree than that of Europe $(82 \%) .{ }^{10}$ For prostate cancer, the most common cancer in men in most western countries, five-year relative survival ranged from nearly $100 \%$ in the United States (2004), ${ }^{23} 92 \%$ in Australia $(2002-2006)^{22}$ to $83 \%$ in Europe (2000-2007), ${ }^{10}$ while the corresponding figure in China for 2003-2005 was 54\%. It is likely that at least part of these differences are due to differences in screening practices, particularly mammography screening for breast can$\operatorname{cer}^{26}$ and prostate-specific antigen testing for prostate cancer, which are more widely used in Western countries than in China. Although one impact of differential screening and diagnostic practices would be to artificially inflate survival estimates through overdiagnosis, it is impossible to quantify 
Table 2. Age-standardized 5-year relative survival (RS, \%): China, 17 registries, patients diagnosed 2003-2005

\begin{tabular}{|c|c|c|c|c|c|c|}
\hline \multirow[b]{2}{*}{ Cancer (sex) } & \multicolumn{2}{|c|}{ All } & \multicolumn{2}{|c|}{ Urban } & \multicolumn{2}{|c|}{ Rural } \\
\hline & No. of patients & RS (95 \% Cl) & No. of patients & RS (95\% Cl) & No. of patients & RS (95 \% Cl) \\
\hline All cancers & 138,852 & $30.9(30.6-31.2)$ & 70,827 & $39.5(39.1-39.9)$ & 68,025 & $21.8(21.4-22.2)$ \\
\hline Males & 81,798 & $26.6(26.2-27.0)$ & 38,845 & $33.9(33.3-34.4)$ & 42,953 & $19.6(19.1-20.1)$ \\
\hline Females & 57,054 & $36.6(36.1-37.1)$ & 31,982 & $45.3(44.7-46.0)$ & 25,072 & $25.6(25.0-26.2)$ \\
\hline Lung & 25,468 & $16.1(15.6-16.6)$ & 15,328 & $19.5(18.8-20.3)$ & 10,140 & $11.2(10.5-11.9)$ \\
\hline Males & 16,847 & $15.4(14.8-16.0)$ & 9,639 & $19.0(18.1-19.9)$ & 7,208 & $10.9(10.0-11.8)$ \\
\hline Females & 8621 & $17.4(16.5-18.3)$ & 5,689 & $20.5(19.4-21.8)$ & 2,932 & $11.8(10.6-13.2)$ \\
\hline Stomach & 20,362 & $27.4(26.7-28.1)$ & 6,081 & $32.5(31.2-33.9)$ & 14,281 & $24.9(24.1-25.8)$ \\
\hline Males & 14,170 & $27.9(27.0-28.8)$ & 4,222 & $31.8(30.2-33.4)$ & 9,948 & $26.0(24.9-27.1)$ \\
\hline Females & 6,192 & $26.5(25.3-27.7)$ & 1,859 & $34.3(32.0-36.9)$ & 4,333 & $22.9(21.6-24.4)$ \\
\hline Liver & 16,816 & $10.1(9.5-10.7)$ & 6,041 & $16.1(15.0-17.2)$ & 10,775 & $6.3(5.7-7.0)$ \\
\hline Males & 12,690 & $10.2(9.5-11.0)$ & 4,595 & $16.1(14.8-17.6)$ & 8,095 & $6.3(5.5-7.3)$ \\
\hline Females & 4,126 & $10.3(9.4-11.4)$ & 1,446 & $16.8(14.9-19.1)$ & 2,680 & $6.8(5.8-8.0)$ \\
\hline Oesophagus & 16,019 & $20.9(20.2-21.7)$ & 2,596 & $19.1(17.4-20.9)$ & 13,423 & $21.2(20.3-22.0)$ \\
\hline Males & 10,879 & $19.9(19.0-20.8)$ & 2,073 & $18.9(17.0-20.9)$ & 8,806 & $20.1(19.0-21.2)$ \\
\hline Females & 5,140 & $23.6(22.3-25.0)$ & 523 & $23.6(19.5-28.7)$ & 4,617 & $23.6(22.2-25.1)$ \\
\hline Colorectum & 11,711 & $47.2(46.2-48.3)$ & 7,940 & $51.2(49.9-52.6)$ & 3,771 & $38.4(36.5-40.3)$ \\
\hline Males & 6,407 & $48.1(46.6-49.6)$ & 4,388 & $51.8(50.0-53.7)$ & 2,019 & $39.3(36.7-42.1)$ \\
\hline Females & 5,304 & $46.2(44.7-47.8)$ & 3,552 & $50.5(48.6-52.4)$ & 1,752 & $37.5(35.0-40.2)$ \\
\hline Female Breast & 9,161 & $73.0(71.2-74.9)$ & 6,805 & $77.8(75.7-79.9)$ & 2,356 & $55.9(51.9-60.3)$ \\
\hline
\end{tabular}

the extent by which this could explain the survival differences without stage-specific survival estimates. Country-specific differences in management, including initial treatment, surveillance and support networks are other likely explanations of these survival disparities.

A recent commentary ${ }^{2}$ highlighted the substantial barriers to healthcare faced by the Chinese population, relating to access to care, fatalism about cancer, and traditional medicine, each of which is likely to provide additional explanations for the survival disparities between China and more developed countries.

We found regional differences in survival for all the common cancers between urban and rural areas, as in a previous study. ${ }^{12}$ The excess hazard of death within 5 years of diagnosis in rural areas was almost $40 \%$, even after correction for the higher background mortality in rural areas. The causes for this marked rural-urban disparity in cancer survival are not fully understood, but the poor quality of cancer care and limited access to health care for patients living in rural China are both likely to be important contributors. In rural China, health service delivery has a three-tier network: village doctors, township health centres and county hospitals. The village doctor acts as a gatekeeper, in that people go to their village doctor when they feel ill, and are either treated or referred to the township health centre or county hospital. Therefore, the level of education of the village doctors is an important determinant of the quality of diagnosis and care provided. In China, only $13 \%$ of rural doctors had a medical qualification at bachelor degree or higher, while the great majority (79\%) only had a secondary technical school qualification. ${ }^{27}$ The situation was even worse in western provinces: $70 \%$ of village doctors did not have any formal medical training, and had received an average of only 20 months of medical training. ${ }^{28}$ Added to this lower skill level of village doctors, or maybe because of, residents in rural areas were less likely to seek medical advice when they felt ill than those in urban areas, ${ }^{29}$ thus limiting the potential for earlier diagnosis and treatment if cancer was suspected. These results provide a strong justification for providing more government-funded health resources and services for cancer in rural areas.

For those cancers where there are limited effective treatment options, an emphasis on primary prevention is required. This is particularly relevant for the four most common cancers in China; lung, liver, oesophagus and stomach. ${ }^{30}$ As tobacco smoking is a well-known cause of these fatal cancers, ${ }^{31}$ strengthening the current tobacco control measures in China should be considered a high priority in any national cancer control plan. ${ }^{30}$ In addition, the link between hepatitis $\mathrm{B}$ and liver cancer $^{32}$ has implications for vaccination and other prevention methods for hepatitis B. The time-lag between reduction in smoking prevalence and reduction in lung cancer incidence is more than 20 years, so this strategy clearly has a long term focus, but it is nevertheless crucial to reduce the burden of cancer for future generations. Even 


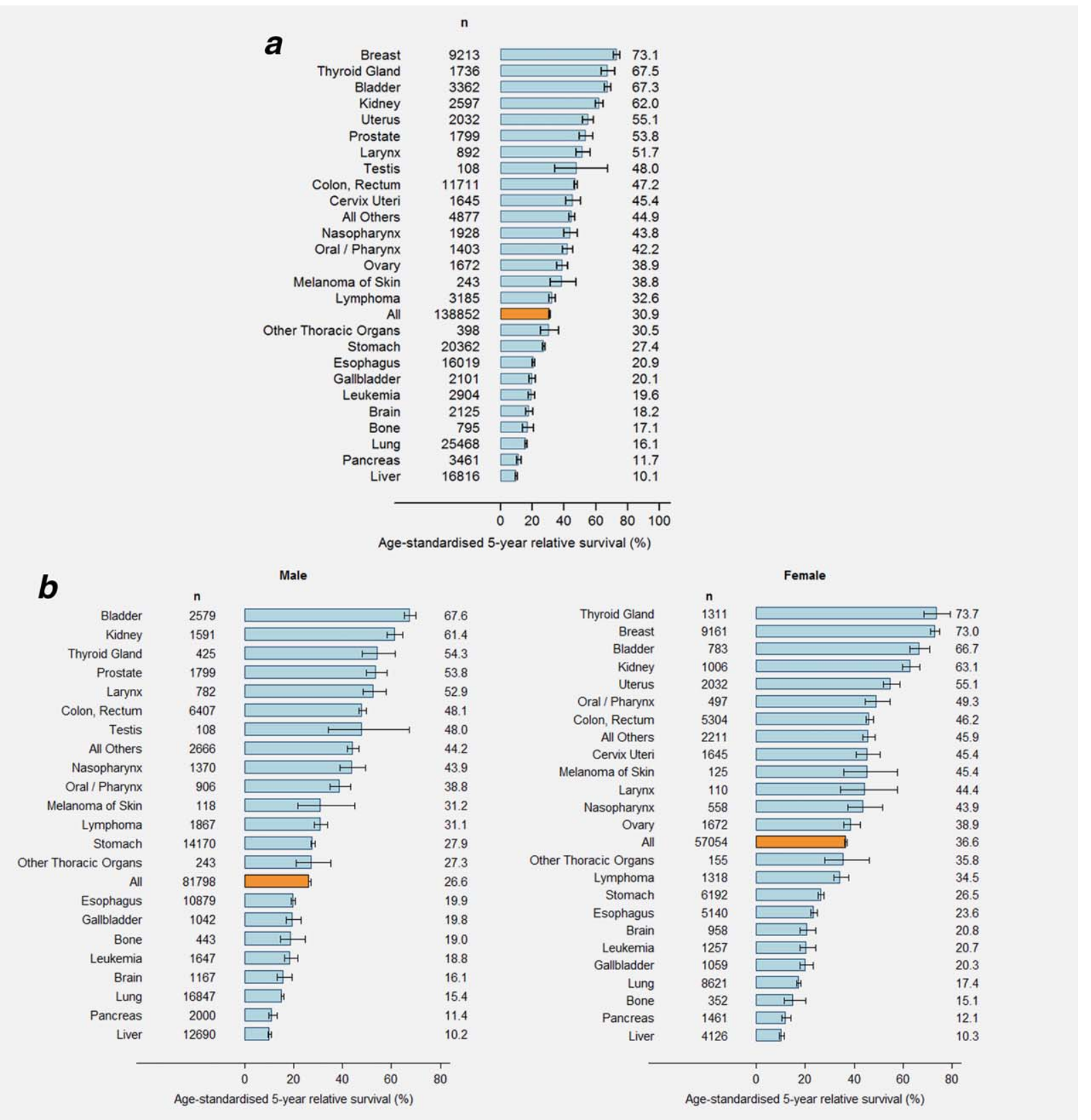

Figure 2. Age-standardised 5-year relative survival by cancer site in China, (a) persons and (b) males and females 2003-2005. [Color figure can be viewed in the online issue, which is available at wileyonlinelibrary.com.]

when such preventive strategies have attained maximal impact, the need for adequate access to effective treatment, including palliative care, will remain. ${ }^{30}$

The optimal level of care for cancer patients is provided by large tertiary hospitals, including pathology, imaging, surgery, radiotherapy, chemotherapy and hormonal therapy. ${ }^{2}$ While cancer patients in China are able to choose their care provider, the vast majority of these tertiary hospitals/centres are located in urban areas, limiting the opportunity for people living in rural communities to obtain appropriate cancer care. In addition, the high cost related with cancer care keeps most rural patients to stay away from such hospitals. ${ }^{29}$ Thus, establishment of an effective primary health care system in rural China, including township health centres and village clinics, and creation of an effective referral network, are both urgently needed ${ }^{33}$ to improve the efficiency and quality of cancer care and to increase the survival of cancer patients who live in rural China.

Previous studies have reported relative survival estimates from four well-established, long-standing cancer registries in China $^{12,34,35}$ (Table 3), three of them urban and one rural. For several cancers for which early diagnosis and treatment makes a difference (Table 3), the magnitude and direction of urban-rural disparities in survival was consistent with what 
All
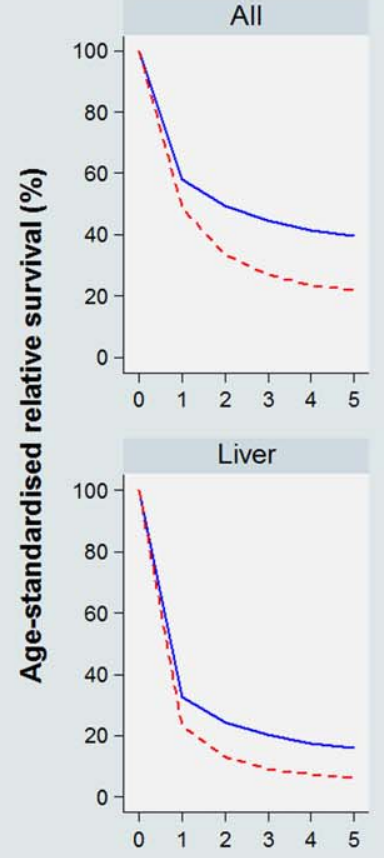

Esophagus

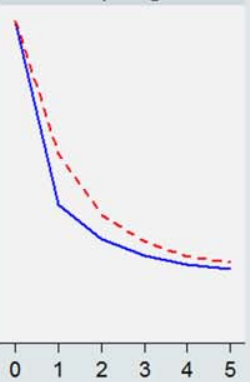

Lung

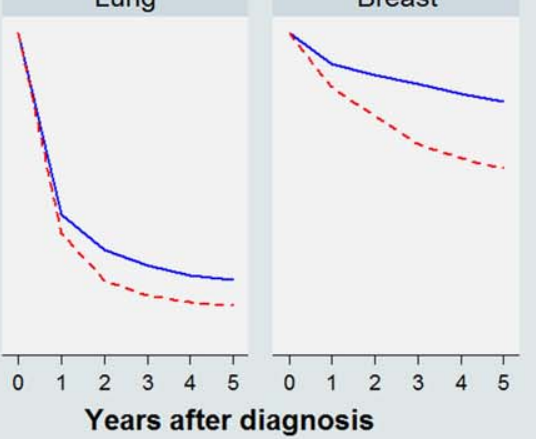

Stomach
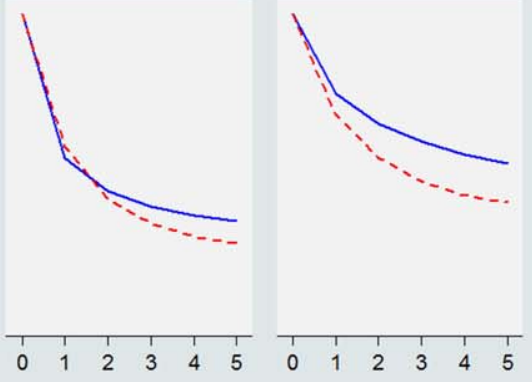

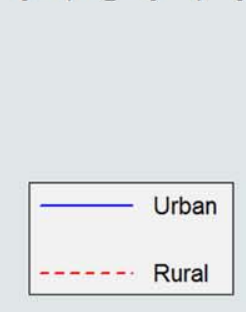

\section{Colonrectum}

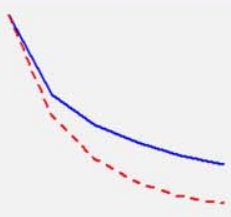

Figure 3. Comparison of age-standardized relative survival curves between urban and rural areas in China for all cancers and six major cancers, persons, 2003-2005. [Color figure can be viewed in the online issue, which is available at wileyonlinelibrary.com.]

we have reported, with survival being markedly lower in the rural area of Qidong than in urban areas of Shanghai, Tianjin and Hong Kong. These studies also provide some evidence of improvement in survival between the 1980s and 1990s, but the magnitude of improvement seemed to be greater within the urban registry (Shanghai) than the rural registry (Qidong). This is also consistent with the marked rural:urban differential in survival that we report here. These previous studies, in conjunction with our later survival estimates, are consistent with the suggestion that the causes of the rural:urban survival differentials in China are long-established. It will therefore take a concerted effort on the part of policy-makers and clinicians to change the cultural, medical and social factors that impact on these differences.

Population-based studies require cancer registries to record suitable information for all cancer patients living in the territory they cover, and can arrange for the survival of those patients to be monitored over time. Incomplete followup and failure to capture all incident cancer cases can bias survival estimates, particularly for cancers with poor prognosis. ${ }^{36}$ To minimize this problem, we excluded data from four cancer registries after investigation of data quality. Underregistration of deaths may lead to over-estimation of longterm survival. ${ }^{37}$ In developing countries such as China, the

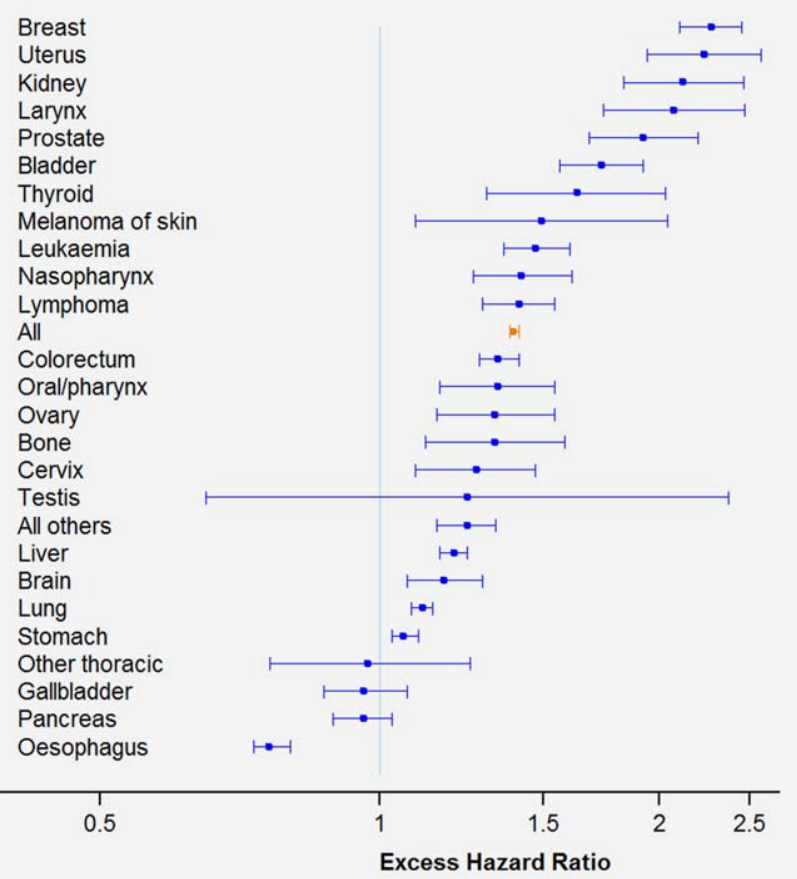

Figure 4. Excess hazard ratios of death within five years in rural versus urban (reference) areas by cancer type in China, cases diagnosed 2003-2005. [Color figure can be viewed in the online issue, which is available at wileyonlinelibrary.com.] 
Table 3. A selection of previously published cancer survival results (by International Agency for Research on Cancer ${ }^{1}$ ) from population-based cancer registries in China

\begin{tabular}{|c|c|c|c|c|c|c|}
\hline \multirow[b]{3}{*}{ Cancer site } & \multirow{2}{*}{\multicolumn{2}{|c|}{$\begin{array}{c}\text { Rural } \\
\text { Qidong }\end{array}$}} & \multicolumn{4}{|c|}{ Urban } \\
\hline & & & \multicolumn{2}{|c|}{ Shanghai } & \multirow{2}{*}{$\frac{\text { Tianjin }}{1991-1999}$} & \multirow{2}{*}{$\frac{\text { Hong Kong }}{1996-2001}$} \\
\hline & 1982-1991 & $1992-2000$ & 1988-1991 & 1992-1995 & & \\
\hline Oral cavity & 34.0 & 36.2 & 52.1 & 63.4 & 64.6 & 60.2 \\
\hline Nasopharynx & 28.7 & 31.2 & 53.3 & 56.0 & 55.3 & 74.6 \\
\hline Colon & 34.5 & 38.9 & 43.5 & 50.6 & 61.4 & 61.3 \\
\hline Rectum & 25.3 & 31.4 & 42.8 & 47.7 & 56.0 & 60.6 \\
\hline Breast & 59.5 & 59.4 & 72.0 & 78.7 & 84.8 & 89.8 \\
\hline Cervix & 37.2 & 39.4 & 51.9 & 60.1 & 62.4 & 76.8 \\
\hline Bladder & 40.1 & 42.9 & 60.9 & 64.3 & 74.6 & 75.5 \\
\hline
\end{tabular}

${ }^{1}$ Cancer survival in Africa, Asia, the Caribbean and Central America edited by Sankaranarayanan R and Swaminathan R. IARC Scientific Publications. no 162. Lyon: IARC; 2011.

death certificates for cancer patients are often inaccurate as to the cause of death. The relative survival approach uses the excess hazard of death, rather than the cause of death for cancer-specific survival. More importantly, however, not all deaths may be reported. ${ }^{38}$ This means that passive follow-up alone may not be sufficient to ascertain all death records in China. This is reflected by the fact that populations covered by the Dalian and Haining registries, with passive follow-up only, had consistently higher survival than in registries that also used active follow-up. To reduce the impact of this, most registries in our study used both passive and active follow-up to provide more accurate survival estimates. In Beijing, where the death surveillance system is much more advanced than in most other regions in China, active followup detected an additional $7.4 \%$ of deaths compared to passive follow-up alone during 2002-2005. ${ }^{39}$ However, if anything, biased under-registration of deaths would result in overestimation (not under-estimation) of the true survival for Chinese cancer patients, so the wide disparity in survival between China and developed countries may well be even greater than reported here.

Most cancer registries in our study are located in areas with relatively high socioeconomic level. In addition, those regions with formal cancer registration systems generally have better health care facilities and general awareness and skills of doctors in relation to cancer (Fig. 1). Therefore, the survival estimates reported here may not be fully repre- sentative of the whole Chinese population, and may actually over-estimate the true overall national survival. A parallel would be registries in the US SEER programme, which until recently covered a somewhat more affluent segment of the US population, and have higher survival than in other areas. ${ }^{9,40}$ On the other hand, the urban-rural survival gap in China could well be even greater than we have reported here, because survival in many regions and provinces (Gansu, Guizhou) that are not covered by this study is likely to be lower.

In spite of this, the survival estimates reported in this study reflect a critical first step in obtaining and reporting accurate and reliable estimates of survival in China. Considerable efforts have been made to improve data quality in each registry, and these results will serve as a baseline for future comparisons and assessment to understand better the overall effectiveness of cancer health care in China, and to provide insights into the areas of greatest need. Clearly, the outcomes for Chinese people diagnosed with cancer are currently poor, especially for people who live in more rural communities. It remains an urgent priority to understand better the reasons why, and to intervene to reduce these internal and international disparities in cancer outcome. More detailed studies of survival, including information on stage at diagnosis and other clinical characteristics, will assist in better understanding of these disparities. ${ }^{41,42}$ Much more research is needed.

\section{References}

1. Chen Z. The Third National Retrospective Sam pling Survey on Causes of Death.ed. Beijing: Peking Union Medical College Press, 2008.

2. Goss PE, Strasser-Weippl K, Lee-Bychkovsky BL, et al. Challenges to effective cancer control in China, India, and Russia. Lancet Oncol 2014;15:489-538.

3. Ferlay J, Shin HR, Bray F, et al. Estimates of worldwide burden of cancer in 2008: GLOBOCAN 2008. Int J Cancer 2010;127:2893-917.

4. Cancer Registry of Norway. Survival of cancer patients: Cases diagnosed in Norway 19681976ed. Oslo: Norwegian Cancer Society, 1980.
5. Black RJ, Sharp L, Kendrick SW. Trends in cancer survival in Scotland 1968-90ed.: Common Services Agency for the Scottish Health Service, Information \& Statistics Division, Edinburgh, 1993.

6. Cutler SJ. International symposium on end results of cancer therapy. Computation of survival rates. Natl Cancer Inst Monogr 1964;15:381-5.

7. Kachuri L, De P, Ellison LF, Semenciw R. Cancer incidence, mortality and survival trends in Canada, 1970-2007. Chronic Dis Inj Can 2013;33: 69-80.
8. Taylor R, Davis P, Boyages J. Long-term survival of women with breast cancer in New South Wales. Eur J Cancer 2003;39:215-22.

9. Coleman MP, Quaresma M, Berrino F, et al. Cancer survival in five continents: a worldwide population-based study (CONCORD). Lancet Oncol 2008;9:730-56.

10. De Angelis R, Sant M, Coleman MP, et al. Cancer survival in Europe 1999-2007 by country and age: results of EUROCARE-5-a population-based study. Lancet Oncol 2014;15: 23-34. 
11. Wang QJ, Zhu WX, Xing XM. Analysis of the incidence and survival of female breast cancer in Beijing during the last 20 years. Zhonghua Zhong Liu Za Zhi 2006;28:208-10.

12. Sankaranarayanan R, Swaminathan R, Brenner $H$, et al. Cancer survival in Africa, Asia, and Central America: a population-based study. Lancet Oncol 2010;11:165-73.

13. Ferlay J, Burkhard C, Whelan S, Parkin DM. Check and conversion programs for cancer registries. (IARC/IACR Tools for Cancer Registries). IARC Technical Report No. 42, 2005.

14. Ederer F, Heise H. Instructions to IBM 650 programmers in processing survival computation, technical, end results evaluation sectioned. Bethesda, MD: National Cancer Institute, 1959.

15. Elandt-Johnson RC, Johnson NL. Survival models and data analysis (Wiley series in probability and mathematical statistics). Indianapolis: Wiley, 1980.

16. Micheli A, Baili P, Quinn M, et al. Life expectancy and cancer survival in the EUROCARE-3 cancer registry areas. Ann Oncol 2003;14(Suppl 5):v28-40.

17. Greenwood M. The natural duration of cancer.ed. London: HMSO, 1926.

18. Corazziari I, Quinn M, Capocaccia R. Standard cancer patient population for age standardising survival ratios. Eur J Cancer 2004;40:2307-16.

19. National Bureau of Statistics of the People's Republic of China. Beijing, 2014. Available at http://www.stats.gov.cn/tjsj/tjbz/ (accessed on April 1, 2014).

20. Dickman PW, Sloggett A, Hills M, Hakulinen T. Regression models for relative survival. Stat Med 2004;23:51-64.

21. Dickman P, Coviello E, Hills M. Estimating and modeling relative survival. Stata J 2012;2012:1-23.
22. Australian Institute of Health and Welfare. Cancer Survival and Prevalence in Australia: Period Estimates from 1982 to 2010ed. Canberra: AIHW, 2012.

23. Howlader N, Noone A, Krapcho M, et al. SEER Cancer Statistics Review, 1975-2010. Bethesda: National Cancer Institute, 2013.

24. Autier P, Boniol M, Hery C, et al. Cancer survival statistics should be viewed with caution. Lancet Oncol 2007;8:1050-2; author reply 3-4.

25. Gatta G, Capocaccia R, Sant M, et al. Understanding variations in survival for colorectal cancer in Europe: a EUROCARE high resolution study. Gut 2000;47:533-8.

26. Fan L, Strasser-Weippl K, Li JJ, et al. Breast cancer in China. Lancet Oncol 2014;15:e279-89.

27. Anand S, Fan VY, Zhang J, et al. China's human resources for health: quantity, quality, and distribution. Lancet 2008;372:1774-81.

28. Wang G, Xu H, Jiang M. Evaluation on comprehensive quality of 456 doctors in township hospitals. J Health Resour 2003;6:72-4.

29. Liu M, Zhang Q, Lu M, et al. Rural and urban disparity in health services utilization in China. Med Care 2007;45:767-74.

30. Chen W, Armstrong BK, Zheng R, et al. Cancer burden in China: a Bayesian approach. BMC Cancer 2013;13:458.

31. Thun MJ, Carter BD, Feskanich D, et al. 50-year trends in smoking-related mortality in the United States. N Engl J Med 2013;368:351-64.

32. Shepard CW, Simard EP, Finelli L, et al. Hepatitis $B$ virus infection: epidemiology and vaccination. Epidemiol Rev 2006;28:112-25.

33. Li C, Yu X, Butler JR, et al. Moving towards universal health insurance in China: performance, issues and lessons from Thailand. Soc Sci Med 2011;73: 359-66.
34. Sankaranarayanan R, Black RJ, Swaminathan R, Parkin DM. An overview of cancer survival in developing countries. IARC Sci Publ 1998:13573.

35. Sankaranarayanan R, Swaminathan R, Jayant $\mathrm{K}$, Brenner $\mathrm{H}$. An overview of cancer survival in Africa, Asia, the Caribbean and Central America: the case for investment in cancer health services. IARC Sci Publ 2011:257-91.

36. Berrino F. The EUROCARE Study: strengths, limitations and perspectives of population-based, comparative survival studies. Ann Oncol 2003; 14(Suppl 5):v9-13.

37. Brenner H, Hakulinen T. Implications of incomplete registration of deaths on long-term survival estimates from population-based cancer registries. Int J Cancer 2009;125:432-7.

38. Yang G, Hu J, Rao KQ, et al. Mortality registration and surveillance in China: history, current situation and challenges. Popul Health Metr 2005;3:3.

39. Yang L, Wang N, Zhu WX, et al. The analysis of the active follow-up study of registered cancer patients between 2002 and 2005 in urban areas of Beijing. Zhonghua Yu Fang Yi Xue Za Zhi 2012; 46:604-6.

40. Merrill RM, Dearden KA. How representative are the surveillance, epidemiology, and end results (SEER) program cancer data of the United States? Cancer Causes Control 2004;15:1027-34.

41. Allemani C, Sant M, Weir HK, et al. Breast cancer survival in the US and Europe: a CONCORD high-resolution study. Int J Cancer 2013;132: 1170-81.

42. Walters S, Maringe C, Butler J, et al. Breast cancer survival and stage at diagnosis in Australia, Canada, Denmark, Norway, Sweden and the UK, 2000-2007: a population-based study. Br J Cancer 2013;108:1195-208. 